\title{
Breakup, transfer, and fusion studies of exotic "halo" nuclei using an active-target time projection chamber.
}

\author{
J. J. Kolata ${ }^{1, a}$, Daniel Bazin, Daisuke Suzuki, and Wolfgang Mittig ${ }^{2}$, and the AT-TPC Collaboration. \\ 1 Physics Dept., University of Notre Dame, Notre Dame, IN 46556-5670 USA \\ 2 NSCL, Michigan State University, East Lansing, MI 48824-1321 USA
}

\begin{abstract}
A program of study of the reaction mechanisms of exotic "halo" nuclei at energies near the Coulomb barrier is described. The unique opportunities presented by the ReA3 reaccelerated beam facility at the NSCL, coupled with an active-target time projection chamber (AT-TPC), will be fully exploited in this program. An approximately half-scale "prototype" AT-TPC has been constructed and is currently undergoing tests. This device has recently been installed on the TwinSol [1] beam line at the University of Notre Dame, and preliminary results of reaction studies using a low-energy ${ }^{6} \mathrm{He}$ beam are reported.
\end{abstract}

\section{Introduction}

The active-target time-projection chamber (AT-TPC) [2] is a dual-functionality device capable of measuring all reaction products generated in inverse kinematics reactions with sufficient resolution to determine the momentum transfer of the reaction. It consists of a large gas-filled chamber, installed in an external solenoidal magnetic field, which simultaneously plays the roles of traditional active target and time projection chamber. The operating pressure of the gas can range from 0.01-1.0 atm depending on the needs of the experimental program. This allows the chamber conditions to be adjusted based upon the energy of the recoil that must be measured, allowing straggling phenomena to provide the only resolution limitation. The design concept (Fig. 1) also includes the possibility of studying reactions with solid targets.

The AT-TPC, when coupled to the ReA3 reaccelerator at the NSCL[3], provides an ideal instrument for studying fusion reactions. The detector can be operated with, e.g., Ar as both the target and detection medium allowing the tracks, momenta, energy loss, and particle species of charged particles to be directly measured. In a fusion event, the recoil energy of the fusion product is low so it will stop after a very short path, too small to determine its characteristics by the trace alone. However the recoil energy can be determined quite precisely after amplification by a Micromegas device [4], with an estimated resolution of $2 \%$ FWHM. Thus the momentum transfer can be directly determined even if the relationship between the energy and the momentum is smeared out by particle evaporation, and so the contribution of "incomplete fusion" can be straightforwardly assessed. Simulations have also demonstrated that the AT-TPC will allow the characteristics of elastic scattering and transfer/breakup reactions to be determined

\footnotetext{
a e-mail: Kolata.1@nd.edu
}

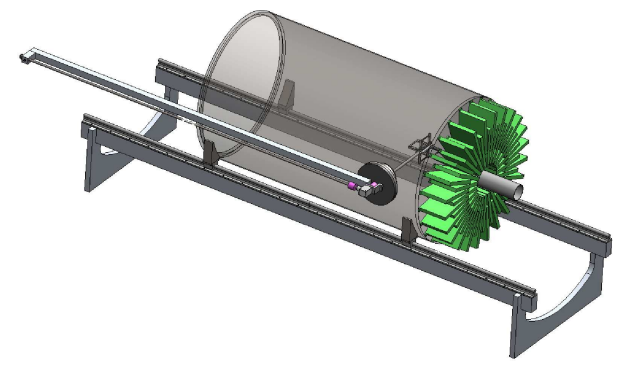

Fig. 1. Conceptual design for the AT-TPC. The active volume is $70 \mathrm{~cm}$ in diameter by $120 \mathrm{~cm}$ in length, and is surrounded by a solenoid [5] having a central field strength of $2 \mathrm{~T}$. The provision for a solid target is illustrated.

with unprecedented resolution in such a low energy experiment. All reaction channels can be measured together and with a very high efficiency. This is an important feature since a complete description of the fusion of exotic "halo" nuclei requires an in-depth understanding of all the important coupled reaction channels of these weakly-bound systems. In addition, the most interesting reactions to study are often those induced by very exotic (hence very weak) beams.

\section{Fusion, Transfer, and Breakup Studies}

In recent years, studies of the reactions of "halo" nuclei such as ${ }^{6} \mathrm{He}$ and ${ }^{8} \mathrm{~B}$ have revealed some interesting effects not seen for more "normal" projectiles (even weaklybound systems such as ${ }^{6,7} \mathrm{Li}$ ). One of the earliest of these was a study of the fusion of the two-neutron-halo nucleus ${ }^{6} \mathrm{He}$ with ${ }^{209} \mathrm{Bi}$ at near- and sub-barrier energies [6]. This experiment, carried out with radiochemical techniques, revealed a rather large enhancement of the fusion cross sec- 
EPJ Web of Conferences

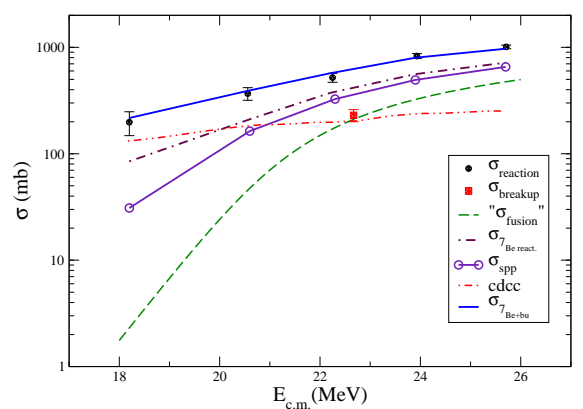

Fig. 2. Total reaction and breakup cross sections for ${ }^{8} \mathrm{~B}+{ }^{58} \mathrm{Ni}$. The various curves represent calculations that are discussed in the text.

tion below the barrier as predicted, e.g., by Dasso and Vitturi [7]. This result has been criticized in light of the possible role played by "incomplete fusion" and particle transfer (discussed further below) in complicating the analysis of the experiment. Furthermore, a study of ${ }^{6} \mathrm{He}+{ }^{238} \mathrm{U}$ fusion, using a fission "trigger", concluded that fusion was not enhanced [8]. (However, there were only two below-barrier points, with large error bars, in this work). As a result, the question of enhanced sub-barrier fusion, and particularly the effect of projectile breakup on the fusion probability, remain controversial.

Several follow-up experiments to the ${ }^{6} \mathrm{He}+{ }^{209} \mathrm{Bi}$ fusion study revealed additional interesting effects. A very intense $\alpha$-particle group resulting from breakup and/or transfer in this system was observed [9] and shown to be consistent with the total reaction cross section obtained from an analysis of elastic-scattering angular distributions [10]. The contributions of one-neutron transfer [11] and two-neutron transfer [12] were measured using neutron-coincidence techniques. Two-neutron transfer is particularly interesting since it was shown [12] to occur to neutron-unbound states in the final nucleus. A similar conclusion was reached in a recent work [13]. It should be noted that, while neutron transfer could be construed as a sort of "incomplete fusion", the observed total reaction cross section [10] leaves little room for other such processes, such as $\alpha$-particle transfer. The ${ }^{6} \mathrm{He}+{ }^{238} \mathrm{U}$ fusion experiment had to be corrected for "transfer-fission" [8], but the radiochemical experiment [6] was insensitive to reaction products resulting from neutron transfer. Finally, direct projectile breakup was studied [14] and shown to be consistent with a coupled-channels calculation [15] assuming a 50\% reduction in the $\mathrm{B}(\mathrm{E} 1)$ computed from a pure ${ }^{4} \mathrm{He}+2 \mathrm{n}$ cluster configuration, in agreement with previous work [16] at much higher energies.

On the basis of the observations above it might be concluded that the reaction mechanisms of neutron-halo nuclei near the Coulomb barrier are now well understood, apart perhaps from the discrepancy in the sub-barrier fusion probability. However, the "fly in the ointment" is the fact that the theoretical study of two-neutron halo systems is quite complicated, requiring 4-body coupled-channels [17] analysis. As a result, most such work is done with cluster models which, as noted above, do not capture all of the physics of the system. Furthermore, calculations of transfer to unbound states require detailed knowledge of continuum states in the target and residual nucleus, which is not readily available for the ${ }^{6} \mathrm{He}+{ }^{209} \mathrm{Bi}$ or ${ }^{6} \mathrm{He}+{ }^{238} \mathrm{U}$ systems. Studies of the much-simpler one-neutron-halo nucleus ${ }^{11} \mathrm{Be}$ are highly desirable. Some work has already been done on the ${ }^{11} \mathrm{Be}+{ }^{209} \mathrm{Bi}$ system and no anomalous effects were observed $[18,19]$.

A significant amount of work has also been done on the reactions of the "proton-halo" nucleus ${ }^{8} \mathrm{~B}$. In particular, the Coulomb breakup of ${ }^{8} \mathrm{~B}$ on ${ }^{58} \mathrm{Ni}$ has been measured at a sub-barrier energy [20]. The results were analyzed by Esbensen and Bertsch [21]. It was found that the standard, first-order Coulex calculation under the usual "pointlike" assumption did not reproduce the experimental data. Instead, it was necessary to account for the extended size of the valence proton wavefunction of the projectile. The center of mass and the center of charge of the projectile cannot be taken to be the same for proton-halo nuclei and the "pointlike" approximation does not hold. In semiclassical terms, the Coulomb-induced breakup cross section at small impact parameter is reduced due to the penetration of the system inside the valence proton orbital. A further reduction is provided by "Coulomb polarization" of ${ }^{8} \mathrm{~B}$. The halo wavefunction has sufficient time to adjust to the proximity of the ${ }^{58} \mathrm{Ni}$ target. Therefore, the proton is more likely to be found at a larger distance from the target, partially shielded from its full Coulomb field by the ${ }^{7} \mathrm{Be}$ core. Finally, it was found that direct proton transfer is also suppressed by Coulomb polarization. The results of this calculation [21] agree rather well with the experimental data. However, a very interesting effect predicted by this calculation could not be verified because the fate of the outgoing proton was not determined in the experiment [20]. Specifically, Coulomb polarization results in the proton being preferentially emitted in the backward direction relative to the ${ }^{7} \mathrm{Be}$ core [22]. The AT-TPC has the capability to directly measure proton emission in the ${ }^{8} \mathrm{~B}+{ }^{40} \mathrm{Ar}$ system and thereby test this prediction.

Recently, the total reaction cross section for ${ }^{8} \mathrm{~B}+{ }^{58} \mathrm{Ni}$ has been determined from elastic-scattering measurements at several energies near to and below the Coulomb barrier, and compared with those for the ${ }^{7} \mathrm{Be}$ core [23]. The strikingly large cross sections observed for ${ }^{8} \mathrm{~B}$ (Fig. 2) are a direct result of the proton halo state. Of particular interest is the relationship between the total reaction cross section and the integrated breakup yield from Ref. [20] which is shown as the square point in Fig. 2. The red curve (dotdot-dashed) in this figure is an extension of the continuumdiscretized coupled-channels (CDCC) calculations of Nunes, Tostevin, and Thompson [24,25] carried out by T.L. Belyaeva [26]. These calculations agree very well with the experimental data at the one energy that has been measured and illustrate the expected excitation function for proton breakup. The dot-dash curve in the figure shows the total reaction cross section for the ${ }^{7} \mathrm{Be}$ core, scaled to remove the geometrical and charge differences between the ${ }^{7} \mathrm{Be}+{ }^{58} \mathrm{Ni}$ and ${ }^{8} \mathrm{~B}+{ }^{58} \mathrm{Ni}$ systems [27]. The sum of these two (the solid curve in Fig. 2) reproduces the observed ${ }^{8} \mathrm{~B}+{ }^{58} \mathrm{Ni}$ data remarkably well. The conclusion is that the total reaction cross sections for this system can be represented as a sum of proton breakup plus the interactions of the ${ }^{7} \mathrm{Be}$ core[28]. 
This leaves little room for proton transfer, as expected from semiclassical arguments based on Coulomb polarization of the halo wavefunction. This is very different from the ${ }^{6} \mathrm{He}$ case, where the same Coulomb polarization argument leads to the conclusion that the neutrons should be preferentially located between the ${ }^{4} \mathrm{He}$ core and the target, thus leading to an increased probability for neutron transfer as observed [12]. The proton transfer cross section for ${ }^{8} \mathrm{~B}+{ }^{40} \mathrm{Ar}$ measured with the AT-TPC could be used to verify this expectation.

The dashed curve in Fig. 2 is the prediction for fusion of ${ }^{8} \mathrm{~B}+{ }^{58} \mathrm{Ni}$ obtained from a barrier penetration model. The parameters of the model $\left(\mathrm{V}_{b}=20.8 \mathrm{MeV}, \hbar \omega=4.4\right.$ $\mathrm{MeV}, \mathrm{R}_{b}=8.9 \mathrm{fm}$ ) were obtained from the real part of the Sao Paulo potential [29] that fits the elastic-scattering data, plus the Coulomb potential (see Ref. [23] for details). They are in good agreement with those obtained from wellknown empirical formulas [30,31]. Attempts have been made to measure this fusion yield. The compound system is the drip-line nucleus ${ }^{66} \mathrm{As}$, which decays predominantly by three-proton evaporation. Unfortunately, this signature was swamped in previous work by background from protons in the incident beam. However, very recently it has been measured using a ${ }^{n a t} \mathrm{Ni}$ target. Remarkably, it turns out that the fusion cross section can be related to that for ${ }^{6} \mathrm{He}+{ }^{209} \mathrm{Bi}$ using an empirical formula for the maximum angular momentum for fusion [32]. The compound system for ${ }^{8} \mathrm{~B}+{ }^{40} \mathrm{Ar}$ is not as exotic, but the fusion signature in the AT-TPC is unique and the resolution should be sufficient to separate complete from incomplete fusion. It will be very interesting to investigate the target dependence of the breakup yield and its effect on the excitation function for fusion.

Finally, it has recently been shown [33] that the "reduced" total reaction cross sections for the proton-halo nucleus ${ }^{8} \mathrm{~B}$ and the two-neutron-halo nucleus ${ }^{6} \mathrm{He}$ follow identical trajectories as a function of the reduced energy, despite differences in the structure, binding energy, and reaction mechanisms of these systems. The reduced cross section is the total reaction cross section divided by $\mathrm{R}^{2}$ and the reduced energy is the $\mathrm{cm}$ energy divided by $\mathrm{V}_{0}$, where:

$$
\begin{gathered}
R=A_{p}^{1 / 3}+A_{T}^{1 / 3}, \\
V_{0}=Z_{p} Z_{T} /\left[A_{p}^{1 / 3}+A_{T}^{1 / 3}\right] .
\end{gathered}
$$

Here, $Z_{p}$ and $Z_{T}$ are the atomic numbers of the projectile and target. This is illustrated in Fig. 3. The AT-TPC could be used to see if this systematic extends to systems involving other halo nuclei.

\section{Simulations of AT-TPC Data}

Properties of the ${ }^{8} \mathrm{~B}+{ }^{40} \mathrm{Ar}$ reaction occurring in the ATTPC have been simulated. Three types of events have been considered: (a) a beam event, i.e., no reaction of the incoming particle (Fig. 4), (b) a fusion event (Figs. 5 and 6) and (c) a break-up event (Figs. 7 and 8). In the fusion event, the recoil energy of the fusion product is low so it will stop

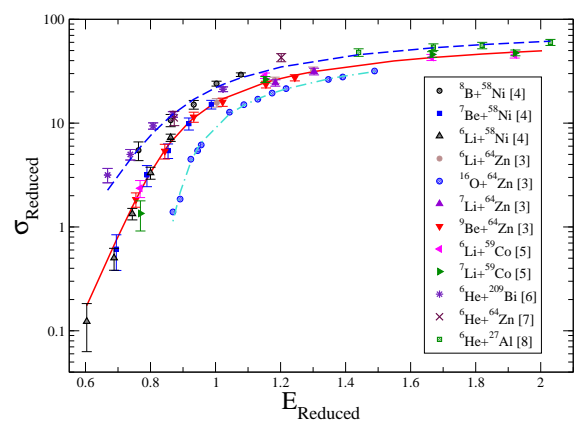

Fig. 3. Reduced total reaction cross sections for a number of systems, taken from the literature. (See Ref. [33])

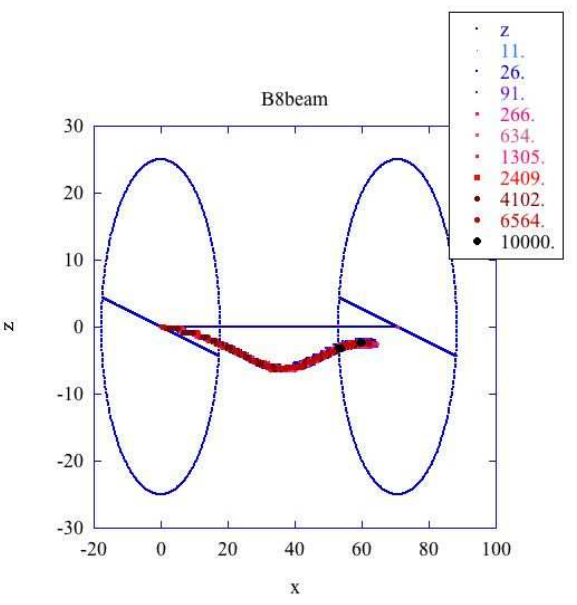

Fig. 4. Trace simulation of a ${ }^{8} \mathrm{~B}$ particle at $25 \mathrm{MeV}$ in Ar gas at $0.05 \mathrm{~atm}$.

after a very short path, too small to determine its characteristics by the trace alone. However the recoil energy can be determined quite precisely. Recent measurements of the charge resolution after amplification by a Micromegas device [4] gave an $\alpha$-particle resolution of 2\% FWHM.

The following parameters were used for the break-up event : ${ }^{7} \mathrm{Be}$ at 25 degrees, $\phi=45$ degrees, and $\mathrm{E}=15 \mathrm{MeV}$. The recoil energy of ${ }^{40} \mathrm{Ar}$ was $1 \mathrm{MeV}$. The proton had an energy of $0.5 \mathrm{MeV}$ and was emitted at 120 degrees in the center-of-momentum frame. As in the fusion case, the recoiling Ar has a low energy and will stop after a very short path, but the recoil energy can be determined quite precisely with a resolution of $2 \%$ FWHM [4]. The proton track is clearly visible and its properties can easily be measured.

The simulations discussed above have shown that the AT-TPC will allow the characteristics of elastic scattering, fusion, and transfer/breakup reactions to be determined with unprecedented resolution in such a low energy experiment. All reaction channels can be measured together and with a very high efficiency. Beams with intensities up to $10^{4} \mathrm{~s}^{-1}$ can be tolerated, based on the drift time in the counter gas. However, it appears very likely that the experimental programs can be carried out at beam intensities of only $1 \times 10^{3}$ $\mathrm{s}^{-1}$ or less, because of the additional flexibility and capabilities of the AT-TPC. This is important since the most 


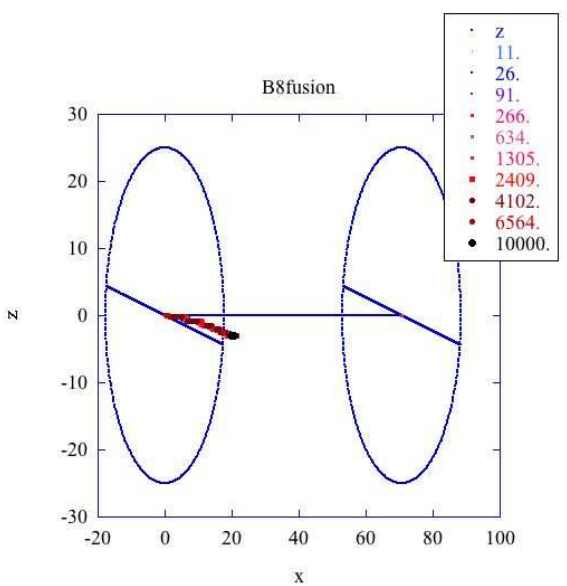

Fig. 5. Trace simulation of a fusion event of ${ }^{8} \mathrm{~B}$ with ${ }^{40} \mathrm{Ar}$.

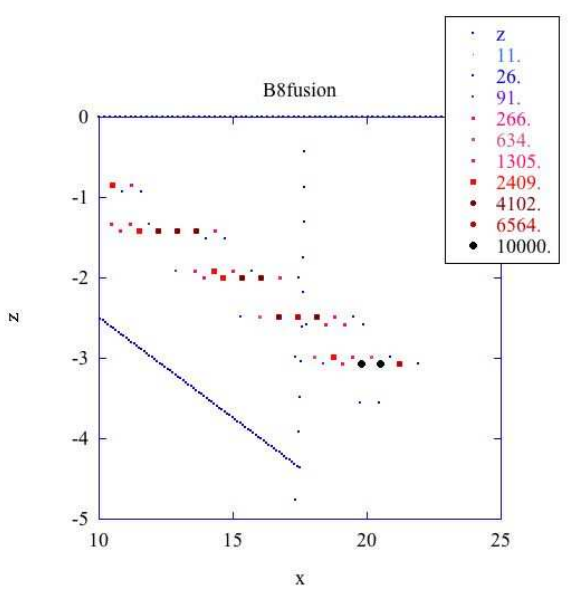

Fig. 6. Zoom of figure 5 .

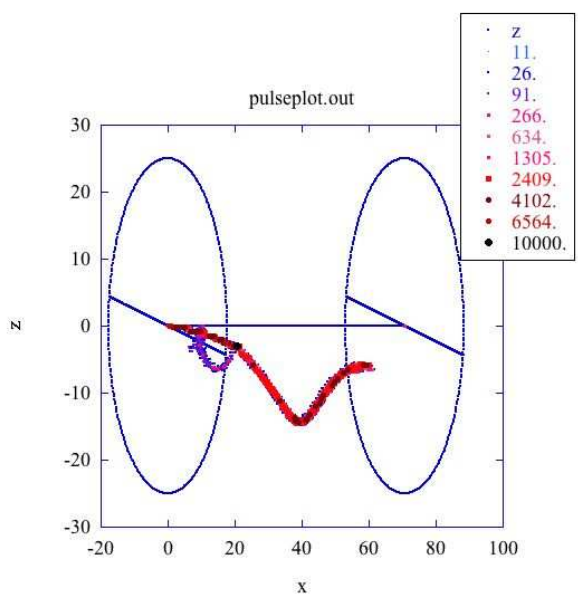

Fig. 7. A break-up event of ${ }^{8} \mathrm{~B}$ into ${ }^{7} \mathrm{Be}$, with one proton emitted at 120 degrees and the ${ }^{7} \mathrm{Be}$ at 25 degrees.

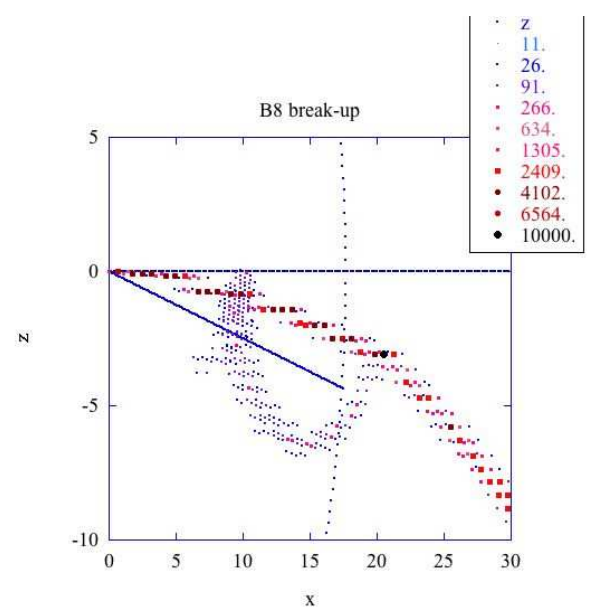

Fig. 8. A zoom of the reaction region of figure 7 .

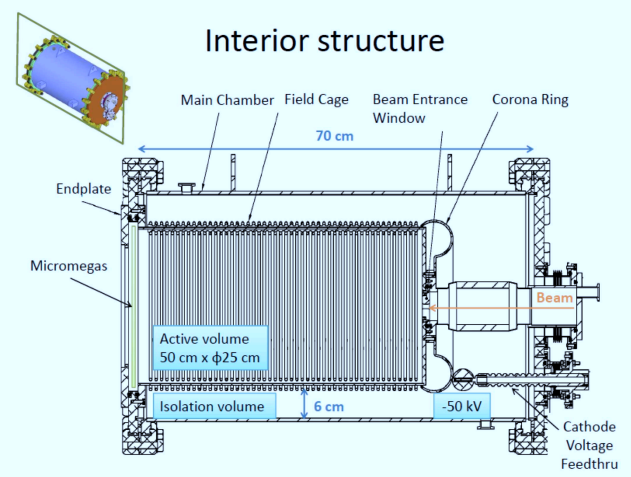

Fig. 9. The prototype AT-TPC

interesting reactions to study are often those induced by very exotic (hence very weak) beams.

\section{The Prototype AT-TPC}

As a first step towards implementation of the AT-TPC, an approximately one-half scale version has been implemented (Fig. 9). The Micromegas board is shown in Fig. 10. The central pad has a diameter of $5 \mathrm{~mm}$. This pad is surrounded by 58 coaxial rings with a width of $2 \mathrm{~mm}$. The first five rings $(\mathrm{r}<13 \mathrm{~mm})$ are divided into 8 sectors, while the outer rings are divided into 4 sectors. This device has recently been commissioned in a run at TwinSol using a 14$\mathrm{MeV}{ }^{6} \mathrm{He}$ beam. (There was no solenoid surrounding the detector). Since there is a limit on the total count rate of the beam that can be tolerated, it was necessary to develop a high-purity, low energy ${ }^{6} \mathrm{He}$ beam, as shown in Fig. 11. This beam was produced using a $29 \mathrm{MeV}{ }^{7} \mathrm{Li}$ primary beam incident on a gas cell containing ${ }^{2} \mathrm{H}$ at 1.5 atm. The cross section at this energy is large compared with that at the lower energy required to directly produce a $14 \mathrm{MeV}{ }^{6} \mathrm{He}$ beam. The resulting radioactive beam was passed through a $5.5 \mathrm{mg} / \mathrm{cm}^{2} \mathrm{CH}_{2}$ foil at the crossover point between the two solenoids. This foil reduces the beam energy to $14 \mathrm{MeV}$ and also purifies it via differential energy loss. In this experiment, the prototype AT-TPC was 


\section{FUSION11}

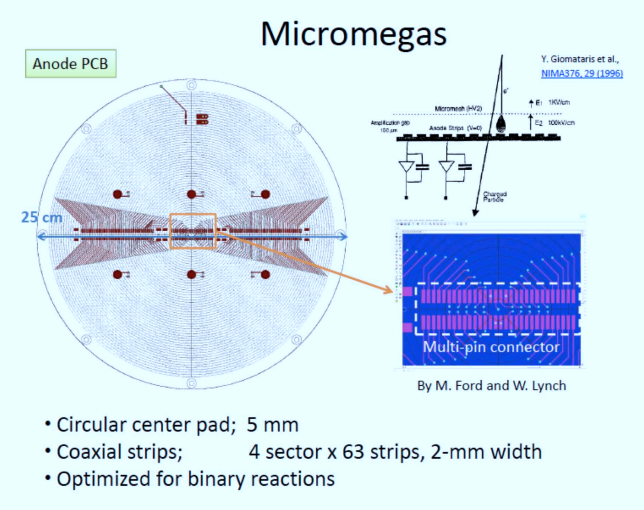

Fig. 10. The Micromegas on the endplate.

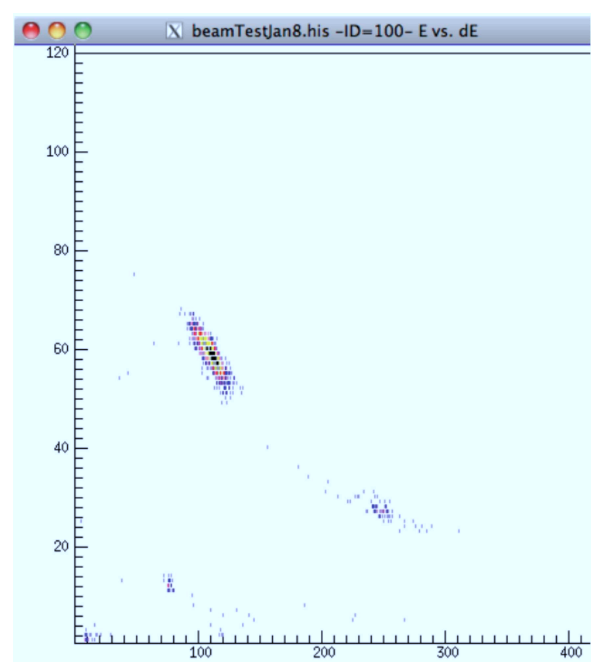

Fig. 11. A "clean" ${ }^{6} \mathrm{He}$ beam at $14 \mathrm{MeV}$. Contaminants of ${ }^{4} \mathrm{He}$ (at $2.9 \%$ of the ${ }^{6} \mathrm{He}$ intensity) and ${ }^{3} \mathrm{H}$ (at $1.4 \%$ of the ${ }^{6} \mathrm{He}$ intensity) are visible.

filled with one atmosphere of ${ }^{4} \mathrm{He}+10 \% \mathrm{CO}_{2}$ and the total beam intensity was limited to approximately $2000-2500$ particles per second to reduce pileup due to the $25 \mathrm{mi}$ crosecond drift time in the counter. The experimental run was completed only one week before the conference so the data have not yet been completely analyzed. However, a selection of interesting events is shown in Figs. 12 and 13.

\section{Conclusion}

The active-target time projection chamber that is being prepared for use with the ReA3 reaccelerated radioactive beam facility at the NSCL will be an extremely useful device for fusion studies of exotic nuclear beams due to its ability to directly image tracks from fusion, elastic scattering, and breakup reactions. All of these reaction processes can be studied at the same time, which is essential for the understanding of fusion. The large solid angle of the device will enable such studies to be carried out with the very weak

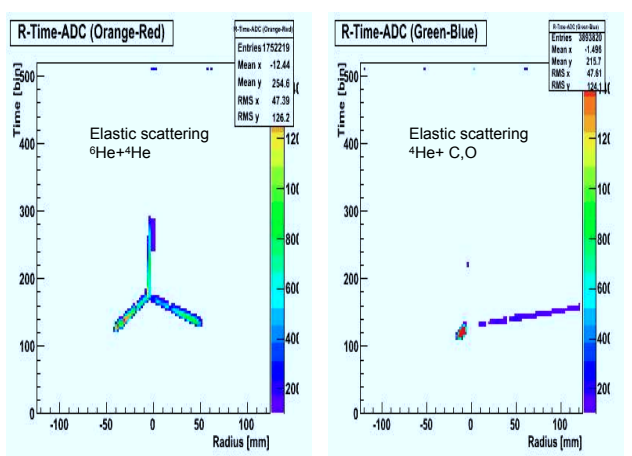

Fig. 12. Sample elastic scattering events from the commissioning experiment.

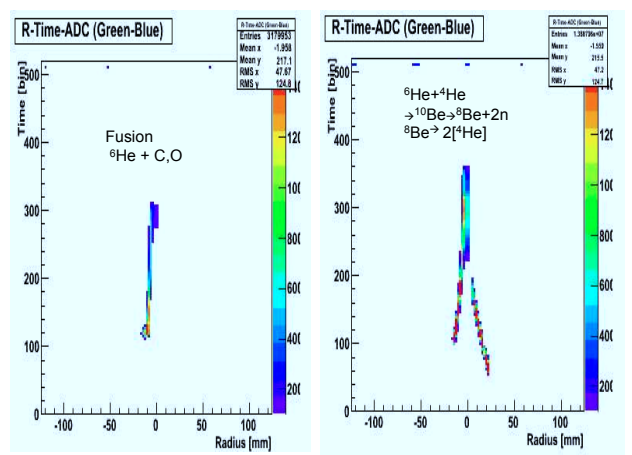

Fig. 13. Sample reaction events from the commissioning experiment..

beams, of the order of $10^{3}$ particles per second, that will be available for the most exotic systems.

A commissioning and physics run with a prototype of this detector has very recently been carried out at the TwinSol facility at Notre Dame. Though the data have not yet been analyzed, initial on-line surveys have shown a wide variety of interesting events. Further studies with additional radioactive beams are planned.

\section{References}

1. F.D. Becchetti, et al., Nucl. Inst. Methods in Physics Research A 505 (2003) 377-380.

2. www.nscl.msu.edu/exp/sr/attpc

3. O. Kester, et al., silver.j-parc.jp/linac10/MO203.PDF

4. www-tpc-paris.cea.fr/talks/TPCHPParis.pdf

5. G. Marshall, Exotic Atoms Conf. 2005, Vienna, Austria (2005)

6. J.J. Kolata, et al., Phys. Rev. Lett. 81 (1998) 4580.

7. C. Dasso and A. Vitturi, Phys. Rev. C 50 (1994) R12.

8. R. Raabe, et al., Nature 431 (2004) 823.

9. E.F. Aguilera, et al., Phys. Rev. Lett. 84 (2000) 5058.

10. E.F. Aguilera, et al., Phys. Rev. C 63 (2001) 061603R.

11. J.P. Bychowski, et al., Phys. Lett. B596 (2004) 26.

12. P.A. DeYoung, et al., Phys. Rev. C 71 (2005) 051601R.

13. A. Chatterjee, etal., Phys. Rev. Lett. 101 (2008) 032701.

14. J.J. Kolata, et al., Phys. Rev. C 75 (2007) 031302R.

15. K. Rusek, et al., Phys. Rev. C 72 (2005) 037603. 
16. T. Aumann, et al., Phys. Rev. C 59 (1999) 1252.

17. T. Matsumoto, T. Egami, K. Ogata, Y. Iseri, M. Kamimura, and M. Yahiro, Phys. Rev. C 73 (2006) $051602(\mathrm{R})$.

18. C. Signorini, et al., Nucl. Phys. A 735 (2004) 329.

19. M. Mazzocco, et al., Eur. Phys. J. A 28 (2006) 295.

20. V. Guimarães, et al., Phys. Rev. Lett. 84 (2000) 1862.

21. H. Esbensen and G.F. Bertsch, Phys. Rev. C 59 (1999) 3240.

22. H. Esbensen, private communication.

23. E.F. Aguilera, et al., Phys. Rev. C79 (2009) 021601R.

24. F. M. Nunes and I. J. Thompson, Phys. Rev. C 59 (1999) 2652.

25. J. A. Tostevin, F. M. Nunes, and I. J. Thompson, Phys. Rev. C 63 (2001) 024617.

26. T. L. Belyaeva, et al., Phys. Rev. C 80 (2009) 064617.

27. P. R. S. Gomes, J. Lubian, I. Padron, and R. M. Anjos, Phys. Rev. C 71 (2005) 017601.

28. E.F. Aguilera, et al., Phys. Rev. C 81 (2010) 011604.

29. L. C. Chamon, et al., Phys. Rev. C 66 (2002) 014610.

30. L. C. Vaz, J. M. Alexander, and G. R. Satchler, Phys. Rep. 69 (1981) 373.

31. R. K. Puri and R. K. Gupta in Heavy Ion Fusion: Exploring the Variety of Nuclear Properties; Proc. Int. Conf.: Padova, 1994, edited by A. M. Stefanini, G. Nebbia, S. Lunardi, G. Montagnoli, and A. Vitturi (World Scientific, Singapore, 1994), p. 319.

32. E.F. Aguilera (this conference).

33. J.J. Kolata and E.F. Aguilera, Phys. Rev. C 79 (2009) 027603 . 